\title{
Análise espaço-temporal das Infecções Respiratórias Agudas em crianças na Região de Saúde de Carajás, Pará
}

\author{
Análisis espacio-temporal de Acute Respiratory Tract Infections in children in the Health \\ Region of Carajás, Pará \\ Spatio-temporal analysis of Infecciones Agudas del Tracto Respiratorio en niños en la \\ Región de Salud de Carajás, Pará
}

Samuel Filipe Lopes Alves ${ }^{1 *}$, Marcelo Gaia Epifane ${ }^{1}$, Erica Silva de Souza Matsumura ${ }^{1}$, Katiane da Costa Cunha 1 .

\section{RESUMO}

Objetivo: Descrever a distribuição espaço-temporal de Infecções Respiratórias Agudas (IRA) em crianças menores de dois anos em uma região do Pará. Métodos: Trata-se de estudo transversal descritivo que utilizou como banco de dados secundário, o Sistema de Informação da Atenção Básica (SIAB). Foram coletados dados da região de saúde de Carajás, Pará, entre 2005 e 2015, acerca de crianças menores de dois anos. Os dados foram tabulados e analisados por estatísticas descritivas com o auxílio do software Tabwin, desenvolvido pelo Departamento de informática do Sistema Único de Saúde (DATASUS). Posteriormente os dados foram mapeados por meio do software Quantum GIS. Resultados: Foram registrados 149.459 casos de IRA em crianças menores de dois anos, com uma média de 13.587 casos por ano. Os meses de março e abril apresentaram as maiores médias mensais; 1368,72 casos/mês e 1278,63 casos/mês e os maiores registros absolutos de casos 15.056 e 14.065 respectivamente. Marabá (36.645) e Parauapebas (20.146) foram os municípios que mais registraram casos de IRA. Conclusão: Conclui-se que os municípios mais endêmicos necessitam de atenção especial quanto aos casos de IRA em crianças menores de 2 anos, sobretudo nos meses de inverno.

Palavras-chave: Infecções Respiratórias, Lactente, Epidemiologia Descritiva.

\begin{abstract}
Objective: To describe the spatio-temporal distribution of Acute Respiratory Infections (ARI) in children under two years of age in a region of Pará. Methods: This is a descriptive cross-sectional study that used as secondary data the Primary Care Information System (SIAB). Data were collected from the health region of Carajás, Pará, between 2005 and 2015, about children under two years old. Data were tabulated and analyzed by descriptive statistics using Tabwin software, developed by the Department of Informatics of the Unified Health System (DATASUS). Subsequently the data were mapped using the Quantum GIS software. Results: 149,459 cases of ARF were recorded in children under two years of age, with an average of 13,587 cases per year. The months of March and April presented the highest monthly averages; 1368.72 cases/month and 1278.63 cases/month and the highest absolute case records 15,056 and 14,065 respectively. Marabá $(36,645)$ and Parauapebas $(20,146)$ were the municipalities that most reported ARI cases. Conclusion: It is concluded that the most endemic municipalities need special attention regarding the cases of ARF in children under 2 years, especially in the winter months.
\end{abstract}

Key words: Respiratory Tract Infections, Infant, Descriptive Epidemiology.

1Universidade do Estado do Pará, Marabá, Pará. *E-mail: samuellopes1212@gmail.com 


\section{RESUMEN}

Objetivo: Describir la distribución espacio-temporal de las Infecciones Respiratorias Agudas (IRA) en niños menores de dos años en una región de Pará. Métodos: Este es un estudio descriptivo de corte transversal que utilizó como base de datos secundario el Sistema de Información de Atención Primaria (SIAB). Se recopilaron datos de la región de salud de Carajás, Pará, entre 2005 y 2015, sobre niños menores de dos años. Los datos se tabularon y analizaron mediante estadísticas descriptivas con la ayuda del software Tabwin, desarrollado por el Departamento de Informática del Sistema Único de Salud (DATASUS). Posteriormente, los datos se mapearon utilizando el software Quantum GIS. Resultados: se registraron 149.459 casos de IRA en niños menores de dos años, con un promedio de 13.587 casos por año. Los meses de marzo y abril presentaron los promedios mensuales más altos; 1368.72 casos/mes y 1278.63 casos/mes y los registros de casos absolutos más altos 15,056 y 14,065 respectivamente. Marabá $(36,645)$ y Parauapebas $(20,146)$ fueron los municipios que más reportaron casos de IRA. Conclusión: Se concluye que los municipios más endémicos necesitan atención especial con respecto a los casos de IRA en niños menores de 2 años, especialmente en los meses de invierno.

Palabras clave: Infecciones del Sistema Respiratorio, Infantes, Epidemiología Descriptiva.

\section{INTRODUÇÃO}

As Infecções Respiratórias Agudas (IRAs) são uma das principais causas de mortalidade e morbidade, sobretudo em crianças. Elas constituem um grupo heterogêneo de infecções respiratórias causadas por diversos antígenos bacterianos e virais e são organizadas em dois subgrupos: Infecções do Trato Respiratório Superior (ITRS) e Infecções do Trato Respiratório Inferior (ITRI) (OMS, 2017). Embora as ITRSs sejam mais frequentes, raramente apresentam risco de vida, já as ITRIs são responsáveis pelas condições mais graves, como pneumonia, tuberculose e bronquiolite (AZEVEDO JVV, et al. 2015).

Segundo a Organização Mundial da Saúde (OMS), existem aproximadamente 1 bilhão de pessoas no mundo que sofrem de problemas respiratórios, sejam eles agudos ou crônicos, tornando-os responsáveis por mais de $10 \%$ de todos os anos de vida ativa e produtiva perdida devido a uma condição, ficando atrás somente das doenças cardiovasculares (GBD, 2015; OMS, 2017). As estimativas a níveis globais das IRAs demonstram a importância dessas condições nas taxas de mortalidade e morbidade, sobretudo em crianças. Por meio da revisão de 89 estudos Rudan I, et al. (2013) estimaram que em 2010, 11,9 milhões de novos episódios graves e 3 milhões de novos episódios muito graves de IRA resultaram em internações de crianças em todo mundo. Também estimaram que ocorreram cerca de 265.000 mortes dentro dos hospitais no ano de 2010 , sendo que $81 \%$ de todas as mortes relacionadas a IRA ocorreram fora dos hospitais.

Outro estudo demonstra que, em 2015, as infecções do trato respiratório inferior (ITRI), entre todas as doenças, obtiveram a quarta maior incidência mundial, com mais de 290 milhões de ocorrências, relacionando-se com um total de 4,9\% das mortes no mundo (VOS T, et al., 2016). As infecções respiratórias também ocupam cinco das 30 causas de morte mais comum em todo mundo, sendo que apenas as ITRIs causam 4 milhões de mortes anualmente (OMS, 2017).

No Brasil, as Infecções Respiratórias Agudas também configuram uma importante problemática em saúde, atingindo especialmente a faixa etária pediátrica. Um estudo desenvolvido a nível continental demonstrou que $40 \%$ das mortes na América Latina em decorrência das IRAs ocorreram no Brasil, totalizando cerca de 32 mil mortes por ano (MARTINS ALO, et al. 2016; PRIETSCH SO, et. al. 2008). Não obstante, Silva MDB, et al. (2012) apontaram que as doenças do trato respiratório foram responsáveis por 1.450 .653 internações, com maior impacto nas crianças, contabilizando $46 \%$ do total das internações em crianças menores de 14 anos.

A faixa etária pediátrica é a principal afetada pelas infecções respiratórias agudas devido a características fisiológicas do trato respiratório. Isso porque nas vias aéreas de um lactente passa o dobro da quantidade de ar que passa no aparelho respiratório de um adulto em repouso, por unidade de peso corporal. Consequentemente, as variações atmosféricas e químicas do ar atingem duas vezes mais o aparelho 
respiratório de uma criança quando comparada a de um adulto no mesmo período (AZEVEDO JVV, et al. 2015).

Os principais fatores socioeconômicos de risco para o desenvolvimento das IRAs em crianças descritos pela literatura são baixo nível de educação parental, baixa renda familiar, tabagismo passivo, amamentação materna exclusiva, interrupção precoce da amamentação, baixo peso ao nascer e desnutrição (LOPES CR e BEZERIN EM, 2009; RODRÍGUES L, et al. 2011; MARTINS ALO, et al., 2016). Fatores climáticos também são constantemente apontados como de risco para as doenças respiratórias, como variações na temperatura, alta umidade relativa do ar e alto índice pluviométrico. Esses fatores colaboram com que a temperatura do ambiente fique abaixo do equilíbrio térmico, o que resulta em um aumento das taxas metabólicas para controlar a temperatura corporal normal. Dessa forma, aumenta-se a necessidade de oxigênio, sobretudo nos lactentes, em que a temperatura corporal é superior aos adultos (AZEVEDO JVV, et al. 2015).

Sabe-se que o Pará possui alguns fatores climáticos que são reconhecidamente de risco para a incidência de doenças respiratórias, como: calor extremo, oscilação térmica, elevada radiação solar e alta umidade relativa do ar (CARVALHO CMM e MARQUES HHS, 2004). Além disso, não foram encontrados trabalhos científicos atuais que ofereçam dados sobre a prevalência de IRA na Região de Saúde de Carajás.

Nesse contexto, o objetivo desse estudo foi analisar a distribuição espaço-temporal dos casos de infecções respiratórias agudas em crianças menores de dois anos entre os anos de 2005 e 2015, a fim de embasar possíveis ações de prevenção e promoção de saúde adaptadas para o comportamento das IRAs na Região de Saúde de Carajás.

\section{MÉTODOS}

Estudo do tipo descritivo, quantitativo, transversal e retrospectivo, que utilizou como banco de dados secundário o Sistema de Informação da Atenção Básica (SIAB), cujos dados são disponibilizados na internet pelo Departamento de Informática do Sistema Único de Saúde (DATASUS). O DATASUS é integrante da Secretaria de Gestão Estratégica e Participativa, que foi institucionalizada pelo artigo 32 do decreto № 7.797, de 30 de agosto de 2012.

Os dados coletados são acerca dos casos de Infecção Respiratória Aguda em crianças menores de dois anos na Região de Saúde de Carajás entre os anos de 2005 a 2015. De acordo com a resolução normativa ํo 259, as Regiões de Saúde são um conjunto de municípios limítrofes que compartilham características culturais, econômicas ou sociais, agrupados a fim de promover a integração das estratégias de ação em saúde. De acordo com dados do Instituto Brasileiro de Geografia e Estatística (IBGE), a Região de Saúde de Carajás possui uma área territorial de $68.302,988 \mathrm{~km}^{2}$ e compreende 17 munícipios da região sudeste do Estado do Pará, que são: Abel Figueiredo, Bom Jesus do Tocantins, Brejo Grande do Araguaia, Canaã dos Carajás, Curionópolis, Dom Eliseu, Eldorado dos Carajás, Itupiranga, Marabá, Nova Ipixuna, Palestina do Pará, Parauapebas, Piçarra, Rondon do Pará, São Domingos do Araguaia, São Geraldo do Araguaia e São João do Araguaia. Em conjunto, os municípios da Região de Saúde de Carajás possuem uma população estimada de 862.729 pessoas para o ano de 2018, com o Índice de Desenvolvimento Humano Municipal (IDHM) variando de 0,528 em Eldorado dos Carajás, a 0,715 em Parauapebas (IBGE, 2019).

Para a obtenção dos dados necessários a pesquisa, no site do Sistema de Informação da Atenção Básica (http://www2.datasus.gov.br/SIAB/index.php?area=04), escolheu-se o Estado do Pará e em seguida fixou-se na linha: Região de Saúde/Município, na coluna: Ano/Mês e no conteúdo: Crianças menores de dois anos com IRA ( $\mathrm{Cr}<2 \mathrm{a}$ c/IRA).

A população estudada correspondeu as crianças com idade até 23 meses e 29 dias que tiveram infecção respiratória aguda nos 15 dias anteriores à visita domiciliar do Agente Comunitário de Saúde (ACS). A população total de crianças menores de dois anos utilizada no cálculo de taxa de incidência foi obtida através do SIAB, somando-se a população menor de um ano de idade com a população entre 12 meses a 23 meses e 29 dias. 
Relativo ao procedimento de coleta dos dados, foi executada a tabulação por meio do software Tabwin, desenvolvido pelo Departamento de Informática do Sistema único de Saúde, com o objetivo de facilitar a tabulação e análise das informações em saúde do portal do DATASUS (SILVA NP, 2009). Quanto a análise dos dados, foi utilizada a descrição dos casos de IRA em crianças menores de dois anos, por meio de medidas estatísticas de dispersão e de termo central. Para a produção dos mapas dos municípios da Região de Saúde de Carajás, foi utilizado o Quantum GIS, um software baseado no Sistema de Informação Geográfica (SIG) que oferece gratuitamente a produção, edição e análise de dados georreferenciados. Esse trabalho seguiu as Diretrizes para o Relato Transparente e Preciso de Estimativas de Saúde (Declaração GATHER), desenvolvido pelo grupo GARTHER convocado pela OMS, a fim de desenvolver boas práticas no relato de estimativas epidemiológicas globais (STEVENS GA, et al. 2017). Todos os recursos necessários a produção e submissão dessa pesquisa foram financiados pelos próprios autores.

Quanto aos aspectos éticos, o presente estudo se baseou na coleta de dados de domínio público e, portanto, não necessita de avaliação pelo Conselho de Ética e da Comissão Nacional de Ética em Pesquisa CEP/CONEP, de acordo com a resolução de nํ510, de 07 de abril de 2016.

\section{RESULTADOS}

Foram registrados na Região de Saúde de Carajás um total de 149.459 casos de IRA em crianças menores de dois anos entre os anos de 2005 e 2015, representando cerca de $10 \%$ de todos os casos de IRA ocorridos no estado do Pará nesses 11 anos estudados. A média anual de casos foi de 13.587 e o ano de 2005 obteve o maior número de casos em todo o período analisado, contabilizando 20.164 casos, sendo que os meses com maiores quantidades de novos casos nesse ano foram março (2433 casos) e abril (1950 casos). $O$ ano de 2015 obteve a menor incidência, contabilizando 6.311 casos de IRA em crianças menores de dois anos. Portanto, observa-se uma tendência decrescente dos casos de IRA ao longo desses 11 anos (Gráfico 1).

Gráfico 1 - Distribuição e comportamento temporal do número de casos de IRAS de 2005 até 2015 ( $\mathrm{N}=1.963 .859$ ).

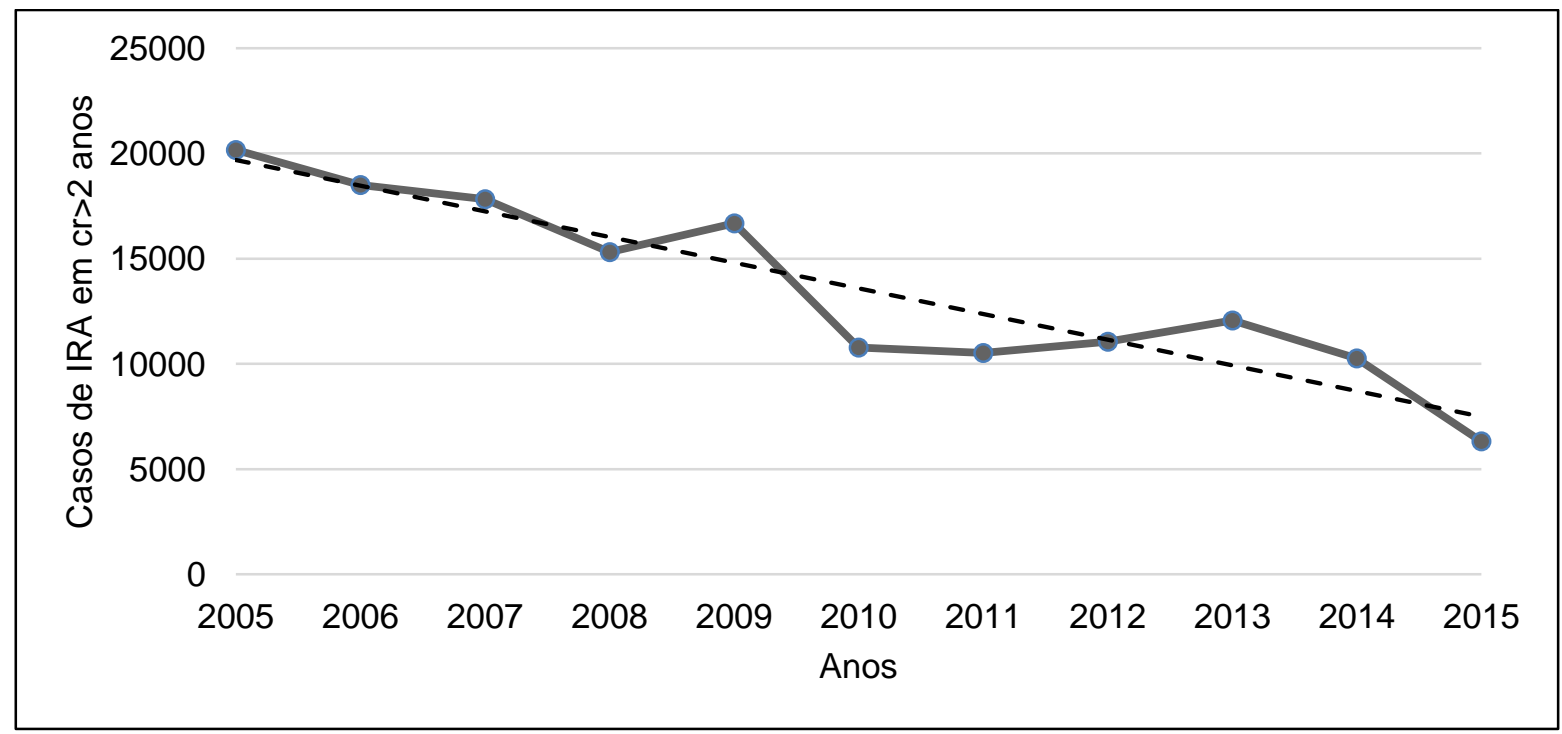

Fonte: SIAB - Datasus, 2019.

Na tabela 1, nota-se que os meses de março e abril apresentaram as maiores médias mensais e os maiores registros absolutos de casos de IRA em crianças menores de dois anos. Os meses de outubro e dezembro apresentaram as menores médias mensais e as menores médias diárias; 32,18 casos/dia e 29,17 casos/dia respectivamente. Por sua vez, os meses de fevereiro e março obtiveram as maiores médias de novos casos por dia de IRA, 43,14 e 44,15, respectivamente (Tabela 1). 
Tabela 1 - Número e estatísticas de Infecções Respiratórias Agudas (IRA) em crianças menores de 2 anos na Região de Saúde de Carajás no período de 2005 a 2015. ( $N=1.963 .859)$.

\begin{tabular}{cccc}
\hline Meses & Média mensal & Desvio Padrão & Total de casos \\
\hline Janeiro & 1111,18 & 267,28 & 12.223 \\
Fevereiro & 1215,72 & 296,94 & 13.373 \\
Março & 1368,72 & 406,46 & 15.056 \\
Abril & 1278,63 & 432,88 & 14.065 \\
Maio & 1207,54 & 448,02 & 13.283 \\
Junho & 1237,36 & 470,18 & 13.611 \\
Julho & 1152,90 & 405,02 & 12.682 \\
Agosto & 1071,63 & 443,91 & 11.788 \\
Setembro & 1024,00 & 345,82 & 11.264 \\
Outubro & 997,63 & 353,27 & 10.974 \\
Novembro & 1017,45 & 393,53 & 11.192 \\
Dezembro & 904,36 & 374,69 & 9.948 \\
Total & 13587,12 & 4638 & 149.459 \\
\hline
\end{tabular}

Fonte: SIAB - Datasus, 2019.

No mapa abaixo, observa-se que houve variações significativas nos casos de IRA entre os municípios constituintes da Região de Saúde de Carajás. O município que apresentou o maior número de casos registrados durante todo o período foi Marabá, com 36.645 casos, tornando-o o sétimo município do estado do Pará com maior frequência de IRA em crianças menores de dois anos. Parauapebas (20.146) e Dom Eliseu (17.813) são respectivamente o segundo e o terceiro municípios com maiores registros de IRA na Região de Saúde de Carajás Em contrapartida, os municípios que obtiveram as menores quantidades de casos registrados de IRA foram Piçarra (548), Canaã dos Carajás (762) e Bom Jesus do Tocantins (1.935), sendo que em Canaã dos Carajás não houve registro de IRA para o ano de 2015 (Mapa 1).

Mapa 1 - Distribuição dos casos de IRA em crianças menores de 2 anos em todo o período de 2005 a 2015. Região de saúde de Carajás, Pará. ( $\mathrm{N}=1.963 .859)$.
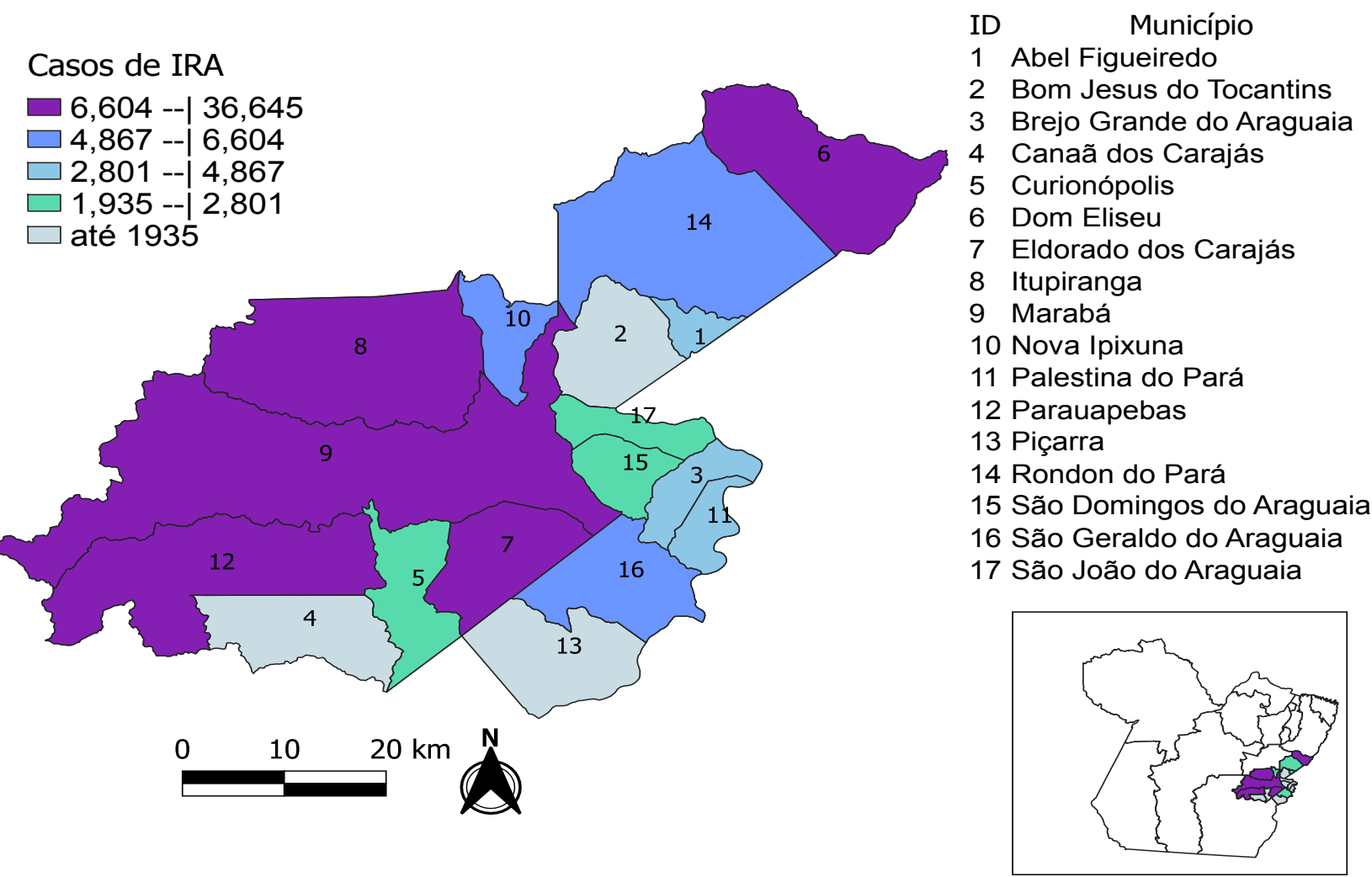

Fonte: SIAB - Datasus, 2019. 
Com relação a taxa de incidência, calculada pela razão entre novos casos de IRA pela população de crianças menores de dois anos em cada município, a tabela 2 aponta que Brejo Grande do Araguaia, Eldorado dos Carajás e Abel Figueiredo apresentaram os maiores indicadores no ano de 2005, ultrapassando $20 \%$. No ano de 2006, nota-se uma leve diminuição de taxa de incidência em Abel Figueiredo e Brejo Grande do Araguaia, enquanto em Eldorado dos Carajás houve um leve aumento que se intensificou no ano seguinte. Em 2007 houve também um aumento da taxa de incidência em Palestina do Pará, que ficou atrás apenas de Eldorado dos Carajás nesse ano. Eldorado dos Carajás, Palestina do Pará e Abel Figueiredo mantiveram-se com as maiores taxas de incidência durante os anos subsequentes, com exceção de 2015, em que Dom Eliseu apresentou a terceira maior taxa de incidência (Tabela 2).

Tabela 2 - Taxa de incidência de IRA em crianças menores de 2 anos. Região de saúde de Carajás, Pará. 2005 até 2015. ( $\mathrm{N}=1.963 .859)$

\begin{tabular}{|c|c|c|c|c|c|c|c|c|c|c|c|}
\hline Município/Ano & 2005 & 2006 & 2007 & 2008 & 2009 & 2010 & 2011 & 2012 & 2013 & 2014 & 2015 \\
\hline Abel Figueiredo & 20,28 & 19,89 & 18,77 & 19,95 & 21,07 & 17,71 & 18,68 & 16,64 & 14,40 & 16,27 & 12,58 \\
\hline Tocantins & 15,65 & 10,71 & 11,85 & 8,51 & 1,12 & 0,02 & 0,05 & 0,12 & 0,05 & 0,29 & 0,27 \\
\hline $\begin{array}{l}\text { Brejo Grande } \\
\text { do Araguaia }\end{array}$ & 20,50 & 20,01 & 17,50 & 8,19 & 10,69 & 7,06 & 9,87 & 10,99 & 12,12 & 11,24 & 9,57 \\
\hline $\begin{array}{l}\text { Canaã dos } \\
\text { Carajás }\end{array}$ & 3,59 & 3,90 & 1,35 & 1,75 & 1,46 & 0,97 & 0,71 & 1,03 & 1,25 & 0,79 & 0,00 \\
\hline $\begin{array}{l}\text { Curionó } \\
\text { Dom El }\end{array}$ & $\begin{array}{l}6,02 \\
8,76\end{array}$ & $\begin{array}{c}5,51 \\
13,76\end{array}$ & $\begin{array}{c}8,14 \\
13,57\end{array}$ & & $\begin{array}{c}5,48 \\
13,98\end{array}$ & $\begin{array}{c}3,84 \\
12,94\end{array}$ & $\begin{array}{c}2,26 \\
12,69\end{array}$ & $\begin{array}{c}0,87 \\
10,56\end{array}$ & $\begin{array}{c}0,38 \\
12,85\end{array}$ & $\begin{array}{c}0,08 \\
11,47\end{array}$ & $\begin{array}{c}0,33 \\
13,55\end{array}$ \\
\hline $\begin{array}{l}\text { Eldorado do } \\
\text { Carajás }\end{array}$ & 20,35 & 21,18 & 27,14 & 24,45 & 24,72 & 23,08 & 20,45 & 17,63 & 16,65 & 14,97 & 13,81 \\
\hline $\begin{array}{r}\text { Itup } \\
\text { Me } \\
\text { Nova }\end{array}$ & $\begin{array}{c}9,16 \\
13,39 \\
8,56\end{array}$ & $\begin{array}{c}10,51 \\
12,66 \\
9,13\end{array}$ & & $\begin{array}{c}10,89 \\
7,44 \\
12,82\end{array}$ & & $\begin{array}{c}10,76 \\
5,54 \\
14,07\end{array}$ & $\begin{array}{c}11,90 \\
2,63 \\
14,57\end{array}$ & & & & $\begin{array}{c}4,16 \\
11,82 \\
6,64\end{array}$ \\
\hline $\begin{array}{l}\text { Palestina do } \\
\text { Pará }\end{array}$ & 19,87 & 13,62 & 22,29 & 14,83 & 17,00 & 19,86 & 18,97 & 17,23 & 14,85 & 16,68 & 15,68 \\
\hline $\begin{array}{r}\text { Paraua } \\
\text { Piça }\end{array}$ & $\begin{array}{c}10,98 \\
0,97\end{array}$ & & & & & & & & $\begin{array}{l}3,63 \\
1,28\end{array}$ & & $\begin{array}{l}6,03 \\
0,10\end{array}$ \\
\hline $\begin{array}{l}\text { Rondon do } \\
\text { Pará }\end{array}$ & 4,83 & 3,77 & 4,37 & 3,17 & 2,91 & 2,64 & 2,02 & 2,15 & 2,17 & 3,01 & 3,00 \\
\hline $\begin{array}{l}\text { São Domingos } \\
\text { do Araguaia }\end{array}$ & 3,27 & 2,94 & 2,65 & 3,52 & 4,69 & 2,68 & 2,10 & 2,58 & 2,22 & 1,28 & 1,61 \\
\hline $\begin{array}{l}\text { São Geraldo do } \\
\text { Araguaia }\end{array}$ & 8,97 & 5,71 & 3,87 & 8,34 & 6,60 & 5,71 & 5,36 & 4,43 & 5,43 & 4,39 & 2,75 \\
\hline $\begin{array}{l}\text { São João do } \\
\text { Araguaia }\end{array}$ & 6,92 & 3,07 & 2,73 & 4,94 & 5,13 & 3,75 & 2,85 & 2,74 & 1,52 & 2,69 & 3,04 \\
\hline Total & $\begin{array}{c}175,1 \\
5 \\
\end{array}$ & $\begin{array}{c}161,4 \\
2\end{array}$ & $\begin{array}{c}172,1 \\
8 \\
\end{array}$ & 146 & $\begin{array}{c}146,8 \\
7\end{array}$ & $\begin{array}{c}131,9 \\
6\end{array}$ & 129,07 & $\begin{array}{c}123,3 \\
5\end{array}$ & $\begin{array}{c}118,4 \\
7\end{array}$ & 112,53 & 99,15 \\
\hline
\end{tabular}

Fonte: SIAB - Datasus, 2019.

De acordo com a Tabela 2, entre os anos de 2005 a 2008, Piçarra, Canaã dos carajás, São Domingos do Araguaia e Rondon do Pará apresentaram as menores taxas de incidência, nunca ultrapassando 5\%. Em 2009, Bom Jesus do Tocantins obteve a menor taxa, fato que se manteve até 2013. Nos anos subsequentes, Curionópolis, Piçarra, Canaã dos Carajás e Bom Jesus do Tocantins obtiveram as menores taxas de incidência (Tabela 2).

\section{DISCUSSÃO}

A diminuição dos casos de IRA na Região de Saúde de Carajás entre os anos 2005 e 2015 encontrada nesse estudo corrobora diversos outros trabalhos realizados no Brasil (SANTOS DAS, et al. 2016). Essa tendência negativa provavelmente é resultado de uma melhoria das condições de vida da população, como aspectos sociais e econômicos. Mas também pode estar associada com uma melhoria da Atenção Básica, 
como uma maior cobertura da Estratégia da Saúde da Família (ESF) durante o período analisado, já que de acordo com Secretaria de Atenção Primária à Saúde, a cobertura da ESF na população da região de saúde de Carajás passou de 19,28\% em julho de 2007 (o dado mais antigo disponível), para 51,19\% em dezembro de 2015 (SAPS, 2019).

Quanto a distribuição mensal dos casos de IRA, percebe-se que os meses com maiores incidências dessas doenças em crianças menores de dois anos coincidem com os meses de maior precipitação e maior humidade relativa do ar, que, na Região de Saúde de Carajás, concentra-se nos meses de novembro até maio (BASTOS TX e PACHECO NA, 2005). Esse achado é consoante a diversos estudos na área, que apontam a relação entre a maior precipitação e maior umidade relativa do ar com aumento da incidência de IRA, sobretudo nos meses de inverno (AZEVEDO JVV, et al. 2015; GINA REPORT, 2006; COELHO-ZANOTI MSS, 2007).

A análise comparativa da taxa de incidência entre os municípios converge para a correlação do fator social como um provável gerador de vulnerabilidade de uma população. Segundo o Atlas de Desenvolvimento Humano no Brasil (2019), no ano de 2010, Parauapebas e Canãa dos Carajás figuraram como os municípios da Região de Saúde de Carajás com melhor Índice de Desenvolvimento Humano Municipal (IDHM), possuindo também baixas taxas médias de incidência de IRA em crianças menores de dois anos, que no total do período analisado correspondeu a 5.46 e 1.52, respectivamente.

A efeito comparativo, os municípios da região de saúde de Carajás que obtiveram os piores IDHM no ano de 2010 foram Itupiranga e Eldorado dos Carajás, que também obtiveram altas taxas de incidência de IRA nos 11 anos de estudo, contabilizando 10.25 e 20.40, de taxa média de incidência de IRA respectivamente. Os resultados são paralelos a de estudos que evidenciaram as determinações sociais na epidemiologia das doenças respiratórias na infância, sobretudo infecciosas, já que a baixa infraestrutura domiciliar propicia um maior risco de contágio e propagação desse tipo de doença (AKERMAN M, et al. 2008; FILHO EBS, et al. 2017).

Além disso, a taxa anual de incidência de IRA para cada município demonstrou uma tendência uniforme de regressão, ao atentarmos para os valores do início e o fim, exceto para Dom Eliseu, no qual a incidência calculada para o último ano foi maior que a do primeiro. A maior parte dos municípios apresentaram anos de alta intercalados entre sequências anuais de queda, logo, ao analisar os valores de início e de fim, pode-se deparar-se apenas com mais uma variação e não uma comprovação cabal de que se trata de uma regressão. Mesmo assim, ao analisar o comportamento temporal na incidência de IRA entre os municípios, pode-se observar uma relativa melhora na eficiência das ações de promoção de saúde desenvolvidas pela atenção primária, através da redução da incidência na maioria dos componentes da Região de Saúde.

Nesse sentido, atenta-se para dois grupos de municípios supracitados que, embora demonstrem uma regressão na incidência, destacam-se como opostos, no sentido de maiores e menores valores de incidência entre todos os componentes da região de saúde. Consoante à análise de Santos DAS, et al. (2017), essa disparidade pode ser relacionada a diferenças na qualidade da assistência à saúde, a qual consiste em ações de promoção de saúde e prevenção da doença, que são desenvolvidas na atenção primária a nível municipal.

\section{CONCLUSÃO}

Embora a quantidade de casos de IRA ter decrescido nos últimos anos analisados, as IRAs ainda atingem a qualidade de vida da população. Com isso, é importante que a educação em saúde seja utilizada para conscientizar a população a respeito dos meses do ano em que é necessário um maior cuidado quanto às infecções respiratórias em crianças menores de 2 anos, sobretudo nos municípios que apresentaram maiores taxas de incidência. Dessa forma, haverá a instrumentalização da população, fortalecendo a participação social no processo de promoção da saúde e prevenção da doença. Vale ressaltar que a falta de uma maior análise estatística de sazonalidade das IRAs e sua correlação com os períodos climáticos do sudeste paraense mostram-se como limitantes desse estudo, de forma que futuros trabalhos possam abordar essa temática com maior profundidade. 


\section{REFERÊNCIAS}

1. ATLAS DO DESENVOLVIMENTO HUMANO NO BRASIL. [Internet]. 2019. Disponível em: http://www.atlasbrasil.org.br/2013/ranking.

2. AKERMAN M, et al. Doenças respiratórias agudas: um estudo das desigualdades em saúde. Cadernos de Saúde Pública, 2008; 24(1): 55-69.

3. AZEVEDO JVV, et al. Influência do clima na incidência de infecção respiratória aguda em crianças nos municípios de campina grande e monteiro, Paraíba, Brasil. Revista Brasileira de Meteorologia, 2015; 30(4): 467 - 477.

4. BERLAN D. Pneumonia's second wind? A case study of the global health network for childhood pneumonia. Health Policy and Planning, 2016; 31(1): i33-i47.

5. BULLA A, HITZE KL. Acute respiratory infections: a review. Bull World Health Organ, 1978; 56: 481-98.

6. CARVALHO CMN, MARQUES HHS. Recomendação da Sociedade Brasileira de pediatria para antibioticoterapia em crianças e adolescentes com pneumonia comunitária. Washington, 2004; 15(6): 1-3.

7. FILHO EBS, et al. Infecções Respiratórias de Importância Clínica: uma Revisão Sistemática. Revista FIMCA, 2017; 4(1): 6-15.

8. GLOBAL BURDEN OF DISEASES (GBD). Global, regional, and national disability-adjusted life-years (DALYs) for 315 diseases and injuries and healthy life expectancy (HALE), 1990-2015: a systematic analysis for the Global Burden of Disease Study. Lancet, 2015; 388: 1603-1658.

9. IBGE. Instituto Brasileiro de Geografia e Estatística. [Internet]. 2019. Disponível em: https://cidades.ibge.gov.br/brasil/pa/panorama.

10. LOPES CR, BEREZIN EM. Fatores de risco e proteção à infecção respiratória aguda em lactentes. Rev Saúde Pública, 2009; 43: 1030-1034.

11. MARTINS ALO. Incidência de infecções comunitárias de vias aéreas inferiores em crianças. Revista Paulista de Pediatria, 2016; 34 (2): 204-209.

12. ORGANIZAÇÃO MUNDIAL DA SAÚDE (OMS). The Global Impact of Respiratory Disease - Second Edition. Sheffield, European Respiratory Society, 2017; 16-20.

13. PRIETSCH SO, et. al. Acute lower respiratory illness in under-five children in Rio Grande, Rio Grande do Sul state, Brazil: prevalence and risk factors. Caderno de Saúde Pública, 2008; 24: 1429-1438.

14. RODRíGUEZ L. Malnutrition and gastrointestinal and respiratory infections in children: a public health problem. International Journal of Environmental Research and Public Health, 2011; 8: 1174-1205.

15. RUDAN I, et al. Global and regional burden of hospital admissions for severe acute lower respiratory infections in young children in 2010: a systematic analysis. Lancet, 2013; 381: 1380-90.

16. SANTOS DAS, et al. Redução de infecção respiratória aguda em crianças menores de dois anos em RondonópolisMT. Revista de Epidemiologia e Controle de Infecção, 2017; 7(1): 40-46.

17. SAPS/MS/Departamento de Saúde da Família - DESF. Relatório de Cobertura da Atenção Básica. [Internet]. 2019. Disponível em: https://egestorab.saude.gov.br/paginas/acessoPublico/relatorios/relHistoricoCoberturaAB.xhtml

18. SILVA MDB, et al. Doença respiratória aguda na criança: uma revisão integrativa. Revista de enfermagem da UFRJ, 2012; 20(2): 260-6.

19. STEVENS GA, et al. Diretrizes para o relato preciso e transparente de estimativas de saúde: a Declaração GATHER. Revista Epidemiologia e Serviços de Saúde, 2017; 26(1): 215-222.

20. VERONESI R, FOCACCIA R. Tratado de Infectologia. 4 ed. Revista e Atualizada. Editora ATHENEU, 2009; 22332236p.

21. VOS T, et al. Global, regional, and national incidence, prevalence, and years live with disability for 310 diseases and injuries, 1990-2015: a systematic analysis for the Global Burden of Disease Study, 2015. Lancet 2016; 388(10053): $1545-602$.

22. GINA REPORT. Global Strategy for Asthma Management and Prevention - Asthma Management and prevention Chapter 4 - The GINA reports, 2006.

23. COELHO-ZANOTI MSS. Uma análise estatística com vistas a previsibilidade de internações por doenças respiratórias em função das condições meteorológicas na cidade de São Paulo. Tese (Doutorado em Ciências Atmosféricas), Universidade de São Paulo - USP, São Paulo, 2007, 178 p. 